\title{
NOUVELLE
}

\section{Origine des cellules ovales hépatiques}

Yannick Laperche
Inserm U841, Équipe Différenciation et régénération hépatiques, Université Paris XII, Faculté de médecine, 51, avenue du Maréchal de Lattre de Tassigny, 94010 Créteil, France.

Yannick.Laperche@créteil.inserm.fr
> Un article, publié récemment dans Gastroenterology, par Oh et al. [1] relance la controverse sur l'origine des cellules ovales hépatiques. Ces cellules, qui prolifèrent dans la région périportale du lobule hépatique, ont été initialement décrites chez le rat dans le modèle AAF/HX associant une hépatectomie $(H X)$ des deux tiers (qui induit un signal de régénération hépatique) à un traitement par le 2acétyl-aminofluorène (AAF) qui prévient la prolifération des hépatocytes résiduels [2].

\section{L'origine hépatique des cellules ovales} Les cellules ovales présentent de nombreuses caractéristiques des hépatoblastes, qui sont des précurseurs hépatiques fœtaux. Elles ont aussi la capacité de se différencier en hépatocytes ou en cellules biliaires et peuvent constituer une voie de secours pour la régénération du foie qui se met en place quand la capacité proliférative des hépatocytes est altérée.

Leur présence a aussi été rapportée chez la souris [3] et chez l'homme, lors d'atteintes hépatiques aiguës ou chroniques, sous le nom de cellules précurseurs ductulaires ou cellules hépatobiliaires intermédiaires [4]. Leur origine reste encore très discutée, mais il est maintenant admis que, dans leur majorité, les cellules ovales résultent de l'expansion de précurseurs qui persistent à l'état de vestige dans le foie adulte au niveau des canaux de Hering [6] (Figure 1). La dédifférenciation d'hépatocytes en cellules ovales semble devoir être écartée, au moins dans certains modèles expérimentaux [5]. Mais voici que l'hypothèse selon laquelle ces cellules pourrait avoir une origine extra-hépatique et dériver de cellules de la moelle osseuse [7], qui n'avait pas pu être confirmée chez le rat [8] et la souris [5], est à nouveau proposée [1].

\section{Cellules ovales:}

\section{une origine médullaire?}

Pour rechercher la présence de cellules ovales issues de la moelle, oh et al. ont utilisé des rats femelles déficientes pour la dipeptidyl-peptidase IV $\left(D P P I V^{-/}\right)$, ayant reçu une greffe de moelle de rats mâles $D P P I V^{+/+}$. Ces animaux ont ensuite été soumis au protocole AAF/HX. Ils ont également été traités, avant ou après la greffe, par la monocrotaline, un alcaloïde de pyrrolizidine, inhibiteur de la prolifération des hépatocytes et des cellules précurseurs de la moelle.

Lorsque la monocrotaline est administrée 4 semaines avant la greffe, et avant hépactectomie et $A A F$, l'étude histochimique du foie révèle des cellules ovales - positives pour OV-6 et I' $\alpha$-fœtoprotéine (AFP) - qui expriment aussi la DPPIV, ce qui démontre leur origine médullaire. L'analyse des cellules non parenchymateuses isolées à partir du foie de ces rats montre que $40 \%$ sont des cellules ovales $\left(\right.$ AFP $\left.^{+}\right)$et que, parmi ces $40 \%$, la moitié expriment à la fois I'AFP et la DPPIV et viennent donc du greffon.

On note que la monocrotaline n'a pas empêché l'accumulation de cellules ovales d'origine intra-hépati- que. Ces cellules ovales ne semblent pas résulter d'évènements de fusion puisqu'elles ont un ou deux chromosomes $X$, dans un rapport de $1 / 2$ qui correspond au rapport des cellules ovales mâles d'origine intra-hépatique (DPPIV ${ }^{-}$) et femelles extra-hépatique (DPPIV ${ }^{+}$).

Lorsque la monocrotaline est administrée après la greffe, aucune cellule ovale DDPIV ${ }^{+}$'est détectée, montrant que la monocrotaline a inhibé la prolifération, la différenciation de cellules ovales issues du greffon ou leur domiciliation vers le foie, tout en n'empêchant pas l'apparition de cellules ovales d'origine hépatique. Les cellules ovales peuvent donc avoir une origine intrahépatique (cellules des canaux de Hering) ou extra-hépatique (cellules de la moelle osseuse).

Mais ces résultats sont en contradiction avec ceux de Menthena et al. [8] et de Wang et al. [5]. Menthena et al. n'avaient pas identifié, chez des rats DDPIV ${ }^{-/-}$, de cellules ovales issues d'un greffon DPPIV ${ }^{+}$en bloquant la prolifération des cellules hépatiques par la rétrorsine, un autre alcaloïde de pyrrolizidine. Il est possible que cet alcaloïde, tout comme la monocrotaline, ait un effet inhibiteur sur les cellules d'origine médullaire, et pas (ou peu) sur les cellules précurseurs intra-hépatiques localisées dans les canaux de Hering. De même, chez la souris tyrosinémique, Wang et al. avaient montré que les cellules ovales qui s'accumulent dans le foie après administration de $\operatorname{DDC}(3,5$ diéthoxycarbonyl-1,4-dihydrocolli- 
dine) ne viennent pas de la moelle osseuse.

À la lumière des travaux de 0 h et al, on peut admettre que le DDC ait aussi un effet inhibiteur sur les cellules médullaires. De plus, on ne peut pas exclure, sur la base des marqueurs utilisés, que les cellules qui prolifèrent dans ce modèle ne soient pas des cellules biliaires matures.

\section{La double origine des cellules ovales} Ainsi, les cellules ovales qui prolifèrent en réponse à une perte hépatocytaire peuvent avoir une origine intra-hépatique ou médullaire. Leur accumulation dans le foie en phase de régénération ne peut intervenir qu'après inhibition de la prolifération des hépatocytes. La controverse sur leur origine extra-hépatique pourrait tout simplement résulter de la toxicité vis-à-vis des précurseurs médullaires des inhibiteurs utilisés dans certains modèles animaux. Dans le modèle AAF/HX, où l'AAF n'est pas métabolisé en dehors des hépatocytes, Oh et al. montrent que des cellules de la moelle et intra-hépatiques parti- cipent dans des proportions équivalentes à l'accumulation de cellules ovales dans le foie. On peut envisager qu'après une nécrose hépatocytaire, par exposition du foie à des composés qui auraient une toxicité non seulement pour les hépatocytes mais aussi pour les cellules des canaux de Hering, des cellules d'origine médullaire deviennent la source majeure de cellules ovales et puissent suppléer les cellules des canaux de Hering pour le repeuplement du foie par des cellules ovales.

\section{Conclusions}

Cet article souligne à nouveau la capacité de cellules médullaires (dont la nature reste à préciser) à se différencier en cellules hépatiques tout en confirmant que ces cellules n'auront un rôle significatif dans le repeuplement du foie que si elles ont un fort avantage sélectif. De plus, il convient de rester très prudent sur la contribution des cellules ovales, quelle que soit leur origine, à la régénération du foie chez des patients atteints d'hépatopathies. Leur différenciation en

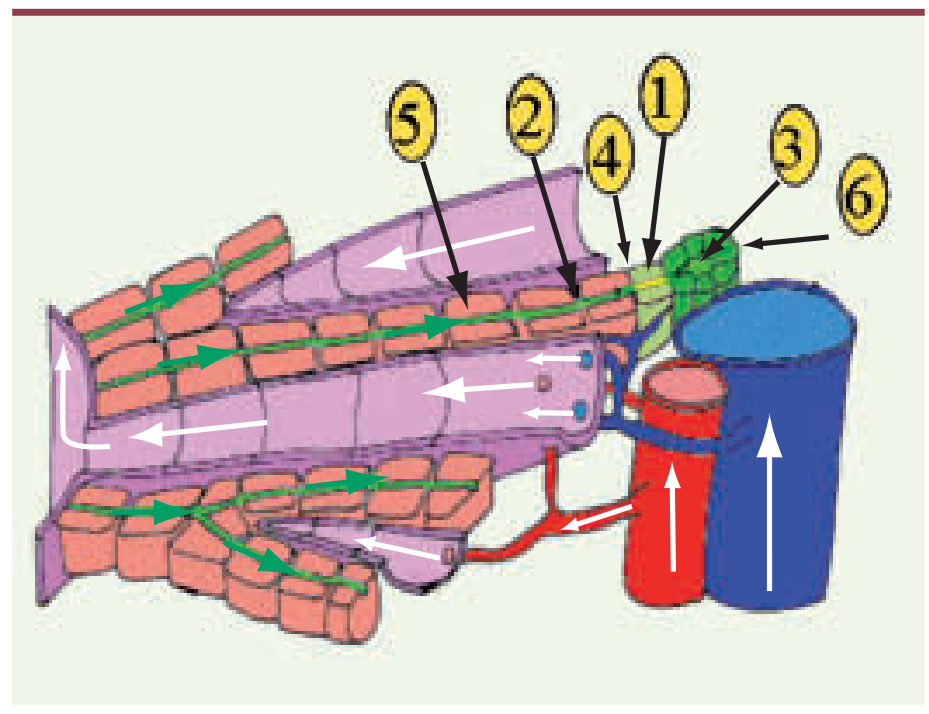

Figure 1. Canal de Hering. Le canal (ou passage) de Hering (1) est un espace entre le canalicule biliaire (2) et le canal biliaire intralobulaire (3). Il est bordé par des cellules (4) qui seraient les cellules précurseurs hépatiques adultes à l'origine des cellules ovales. Ces cellules sont situées à l'extrémité des travées hépatocytaires (5) au contact d'hépatocytes et de cellules bilaires (6) (d'après [11]). hépatocy-

reste incertain. Leur accumulation pourrait aussi être le reflet d'une différenciation altérée et contribuer au développement de la fibrose portale par la sécrétion de facteurs fibrogéniques [10]. $\diamond$

Origin of liver murine oval cells

\section{RÉFÉRENCES}

1. Oh SH, Witek RP, Bae SH, et al. In vivo differentiation of rat liver oval cells into hepatocytes. Gastroenterology 2007 ; 132 : 1077-87

2. Evarts RP, Nagy P, Nakatsukasa H, et al. In vivo differentiation of rat liver oval cells into hepatocytes. Cancer Res $1989 ; 49$ : 1541-7.

3. Petersen BE, Grossbard B, Hatch H, et al. Mouse A6-positive hepatic oval cells also express several hematopoietic stem cell markers. Hepatology 2003 ; $37: 632-40$.

4. Roskams, T. (2003) J Hepatol 39, 431-434

5. Wang X, Foster M, Al-Dhalimy M, et al. The origin and liver repopulating capacity of murine oval cells. Proc Natl Acad Sci USA 2003; 100 (suppl 1) : 11881-8.

6. Theise ND, Saxena R, Portmann BC, et al. The canals of Hering and hepatic stem cells in humans. Hepatology 1999 ; 30 : 1425-33.

7. Petersen $B E$, Bowen WC, Patrene KD, et al. Bone marrow as a potential source of hepatic oval cells. Science $1999 ; 284$ : 1168-70.

8. Menthena A, Deb N, Oertel M, et al. Bone marrow progenitors are not the source of expanding oval cells in injured liver. Stem Cells $2004 ; 22$ : 1049-61.

9. Falkowski 0, An HJ, lanus IA, et al. Regeneration of hepatocyte buds in cirrhosis from intrabiliary stem cells. J Hepatol 2003 ; 39 : 357-64.

10. Clouston AD, Powell $\varepsilon \varepsilon$, Walsh MJ, et al. Fibrosis correlates with a ductular reaction in hepatitis C: roles of impaired replication, progenitor cells and steatosis. Hepatology $2005 ; 41$ : 809-18.

11. Marieb EN. Anatomie et physiologie humaines. Bruxelles : Édition De Boeck, 1999.

ces foies fibreux au sein d'un environ$\mathrm{nement}$ infla $\mathrm{m}$ matoire n'a pas été établie. Tout au plus peuventelles être à l'origine de cellules avec un phénotype intermédiaire [9] dont le devenir
ILLUSTRATIONS DES ARTICles (vignettes): p. 502: hématies et plaquettes (photo Michel Depardieu - (C) Photothèque Inserm) - p. 509: muqueuse du duodénum (photo Rafael Oriol - (c) Photothèque Inserm) - p. 515 : adipocytes en culture (photo Michel Depardieu - (C) Photothèque Inserm) - p. 519: Papilionacées (๔ photo Pierre Rustin) - p. 526: représentation tridimensionnelle de la queue cytosolique de gp9lphox (๔) photo MarieJosé Stasia) - p. 547 : œuf humain âgé de 2 jours après fécondation in vitro (photo Jacques Testart (C) Photothèque Inserm) - page 553 : coupe sagittale de cerveau humain (photo Stéphane Lehéricy (c) Photothèque Inserm). 


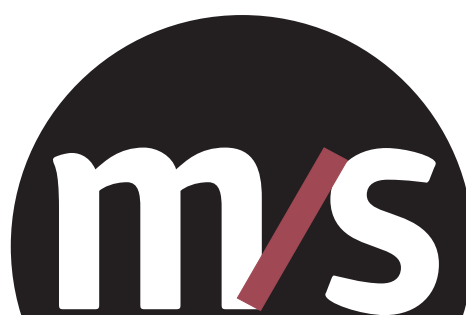

MEDECINE SCIENCES

\section{> Depuis 20 ans, grâce à $m / s$, vous vivez en direct les progrès des sciences biologiques et médicales}

> Des articles rédigés par des médecins et des chercheurs reconnus sur la scène internationale qui posent avec rigueur les bases des débats scientifiques.

> Des synthèses, éditoriaux, dossiers techniques et analyses toujours replacés dans leur contexte pour que l'information soit la plus exacte, intelligible et objective.

avec les articles de référence de M/S

\section{Chaque jour,} sur www.medecinesciences.org
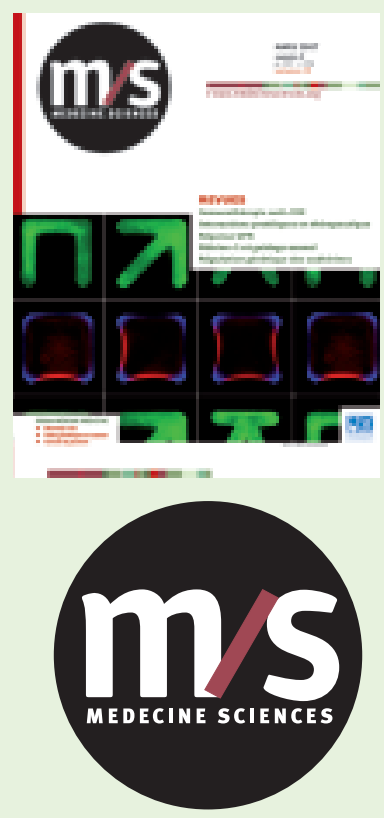

Je souhaite m'abonner à $M / S$ :

Nom : .

Adresse :

Code postal

Pays :

$\varepsilon$-mail-obligatoire :

$$
\begin{array}{cc}
\multicolumn{2}{c}{\text { Particuliers }} \\
\text { Papier } & \text { Électronique } \\
+ & \text { seul } \\
\text { Électronique } &
\end{array}
$$

France $\square 168 € \square 118 €$

UE + autres

$\square 220 €$

$\square 118 €$
Médecine/Sciences

est indexé dans

\section{PubMed/Medline}

Current Contents, série Life Sciences

EMBASE/Excerpta Medica

PASCAL

CABS

BIOSIS

> La dimension humaine privilégiée, avec l'analyse des retombées diagnostiques, thérapeutiques, la prévention et l'éthique liées aux nouvelles avancées.

> Un panorama clair et concis de l'actualité scientifique: des nouvelles, des brèves, des données chiffrées, des repères et perspectives pour qu'aucun fait significatif ne vous échappe.

Je choisis l'abonnement :
Tarifs d'abonnement pour M/S - 2007

\section{Abonnez-vous}

\section{à Médecine/Sciences}

Mon règlement :

$\square$ Parmail edk@edk.fr

Uniquement pour les paiements par carte bancaire

$\square$ Par fax en envoyant ce bulletin au 0155641394

Uniquement pour les paiements par carte bancaire

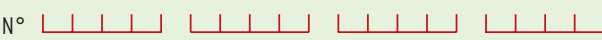
Date d'expiration $L \perp\llcorner\perp \perp$ Signature :

$\square$ Par chèque à l'ordre de Médecine/Sciences, en envoyant ce bulletin à :

Éditions EDK

2, rue Troyon

92316 Sèvres Cedex, France

Pour recevoir une facture, cochez cette case $\square$

Tarifs Canada-USA-Mexique :

Contactez

Médecine/Sciences

500 , rue Sherbrooke Ouest,

bureau 800, Montréal, Québec H3A 3C6, Canada medecine.sciences@bellnet.ca

* Joindre un justificatif

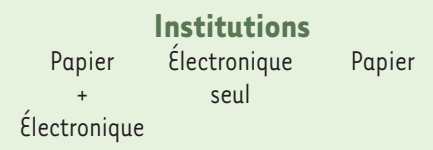

$\square 375 € \quad \square 235 €$

$\square 455 €$ $\square 365 €$

$\square 433 €$
Étudiants* Papier Électronique $\stackrel{+}{\stackrel{+}{\text { Electronique }}+}$

$\square 78 €$ $\square 112 €$

$$
\text { seul }
$$

$\square 62 €$

$\square 62 €$
Enseignants* Papier Électronique $\stackrel{+}{+}$ Électronique

$\square 112 € \quad \square 86 €$

